\title{
A Steady-State Thermal Gradient Generator for Material Characterization Using an Induction Heating System
}

\author{
Veyis Gunes, Jean-Yves Botquélen, Gwenaël Corbel, and Philippe Lacorre
}

\begin{abstract}
Mobility/Diffusion of ions can occur mainly in two situations. The first one when a potential difference is applied to electrodes of an electrochemical device as in batteries for instance (i.e., charge/discharge). In the second one, the diffusion process is driven by the thermal gradient existing within the material. This paper deals with the design of a controlled Thermal Gradient Generator (TGG) which opens the way to thoroughly study the effect of temperature difference on the chemical diffusion and mechanical resistance of shaped samples (e.g., a pellet, a rod). This TGG uses a combination of two susceptors involving the control of their respective temperatures. One is controlled by adjusting the power of a high frequency generator which supplies an induction heating coil. The other is controlled, mainly, by the displacement of the sample/susceptors set through this same coil, with a motorized stage. The operation and control parameters are defined within a dedicated user-friendly software application. During the whole experiment, the two temperatures can be varied while the inner thermal gradient of the sample is kept constant, as long as these temperatures are high enough.
\end{abstract}

Index Terms - Computerized instrumentation, Eddy currents, Induction heating, Materials characterization, Nondestructive testing, Programmable control, Thermal gradient.

\section{INTRODUCTION}

$\mathrm{T}$ THERMAL gradient devices are useful tools to study material properties such as chemical diffusion, thermal conductivity, temperature resistance, ageing, etc. The purpose of the experimental device described in this paper is to apply a controlled thermal gradient to solid samples (a few millimeters thick and about $1 \mathrm{~cm}^{2}$ as cross-section) at high temperatures (up to $1000^{\circ} \mathrm{C}$ and beyond).

In our laboratory team, the studied samples were typically ceramics that undergo ionic diffusion and exsolution when heated up. After applying a thermal gradient across a sample in a nondestructive way, the final goal was to study the microstructures generated by the gradient on and inside the

V. Gunes is with the Institut des Molécules et Matériaux du Mans, Le Mans University, 72085 Le Mans, France (e-mail: veyis.gunes@univlemans.fr).

J.Y. Botquélen, G. Corbel and P. Lacorre are with the Centre National de la Recherche Scientifique, assigned to the Institut des Molécules et Matériaux du Mans, Le Mans University, 72085 Le Mans, France (e-mail: respectively, jean-yves.botquelen@univ-lemans.fr, gwenael.corbel@univ-lemans.fr and philippe.lacorre@univ-lemans.fr). ceramic through characterization techniques such as X-ray diffraction, SEM (Scanning Electron Microscope) or FIBSIMS (Focused Ion Beam-Secondary Ion Mass Spectrometry).

More generally, the TGG presented here can be used to apply any temperature/time controlled gradient at moderate to high temperature between opposite faces of any type of solid insulators or semiconductors, may them be inorganic or polymers. For instance, this device would be suitable for the study of ionic thermomigration in solids (e.g. in memristors, as in Shin et al.[1]) or for the optimization of the thermal treatment of thin films, as in Metting et al.[2] (these are recent studies using more loosely controlled or conventional heating systems). The current TGG is designed to operate in air, but would perform in controlled atmospheres or vacuum too. It allows nondestructive testing.

The main concern of the study is to validate the use of an induction heating system to automatically control and stabilize both the temperature on one side of the sample (e.g., the lower face) and the temperature differential with respect to the other side of the sample, thus allowing a steady-state thermal gradient. This controlled gradient at high temperatures constitutes the novelty of this work. Heating has to be located on small surfaces and temperatures must be measured on both sides. The temperatures of both faces must be as homogeneous as possible in order to have a uniform gradient throughout the section of the sample.

Bober et al. [3] introduced an induction heating system which is built in a high-vacuum, water-cooled recipient. This setup is well suited to higher temperatures, such as $2577^{\circ} \mathrm{C}$ $(2850 \mathrm{~K})$, but the gradient is adjusted manually, without an automatic control. Furthermore, the system is more expensive.

A patented system is described by Pontillon et al. [4]. It consists in a radial (as opposed to axial in our case) gradient at temperatures up to $2000^{\circ} \mathrm{C}$, but it allows a single temperature set point (along the axis of the cylindrical sample). External temperature is fixed by choice of the insulator (depending on its nature and geometry), and chosen by simulation at a temperature of $350^{\circ} \mathrm{C}+/-50^{\circ} \mathrm{C}$, without any attempt of automatic control.

A paper by Shihab et al. [5] describes a heating system by pulsed laser excitation, which can be used only on thin layers (typically $50 \mathrm{~nm}$ thick) of magnetic materials showing a hysteresis cycle, in order to get a thermal differential $(\Delta \mathrm{T})$ of a dozen degrees induced by the laser spot. 
Another patent application, from Obbard et al. [6], describes a device which can establish a thermal gradient within living tissue or an ice core. Two heat sinks are used, each maintained by a control mechanism at a different temperature. Each heat sink is in contact with a thermal conductor, the two conductors being set on two opposite faces of the sample. Thus, an axial thermal gradient is maintained within the sample. However, the use of heat sinks limits applications to temperatures below room temperature.

The solution adopted in our device consists in a single induction coil heating up two susceptors, one on each sample face. Susceptors can be the same if the sample is thick enough to induce a large enough thermal gradient when shifted above or below the coil center, or have different thicknesses in order to increase the gradient. A typical chosen configuration is an upper susceptor with double thickness relative to the lower one, e.g. 300 and $150 \mu \mathrm{m}$, respectively.

The temperature of the lower face and the temperature differential $\Delta \mathrm{T}$ (therefore, the temperature gradient) are regulated by two independent control loops. Compared to a heating system with two heating resistors, our solution has the following advantages:

1) it avoids thermal bridges which inevitably degrade homogeneity in temperatures of the two susceptors (as there are no wires connected to effectors and which could sink-out heat at contact points or contact surfaces).

2) it makes it possible to simulate quenching or quasiquenching more efficiently (with resistors, the thermal bridges would slow down the cooling of the susceptors),

3 ) it is more suitable for thermal cycling and fatigue investigations since it allows higher temperature rise times, as explained by Liu [7].

4) Finally, our solution is much less expensive than two heating systems, because even with heating resistors, the two heating systems would have to be controlled systems that are indeed the most expensive part in such a device.

This article is organized as follows. We first study a manual procedure of the proposed TGG and its preliminary calibration (Section II). Then, the automatic control of the TGG is justified and presented in Section III. Section IV introduces the control software and equipment. This is completed by a validation Section V, with some results. Finally, a conclusion is drawn and some prospects are proposed in Section VI.

\section{MANUAL TGG OPERATION}

Lets $\mathrm{Th}$ and $\mathrm{Tl}$ be the stabilized temperatures of the upper (higher) and lower susceptors, respectively. Let $\mathrm{P}$ be the mass power dissipated by eddy currents in a sheet/pellet (induced from an induction coil, see Figure 1).

Under some conditions (mainly, uniform material, without skin effect), as explained by Nickelson[8], $\mathrm{P}$ is given by:

$$
P=\frac{\pi^{2} B^{2} d^{2} f^{2}}{6 \rho D},
$$

where,

$\mathrm{P}$ is the power lost per unit mass $(\mathrm{W} / \mathrm{kg})$,

$\mathrm{B}$ is the magnetic flux density (T), $\mathrm{d}$ is the thickness of the sheet (m),

$\mathrm{f}$ is the frequency $(\mathrm{Hz})$,

$\rho$ is the resistivity of the material $(\Omega \mathrm{m})$, and

$\mathrm{D}$ is the density of the material $\left(\mathrm{kg} / \mathrm{m}^{3}\right)$.

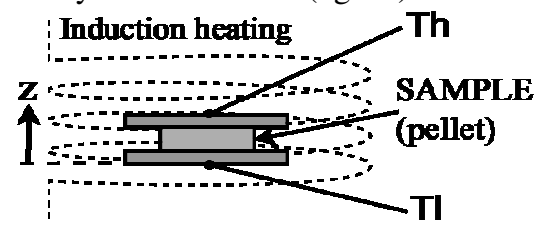

Fig. 1: The induction heating system: the two susceptors, heated up by eddy currents, impose a thermal gradient to a sample. Susceptors together with sample constitute the mobile set. The coil is represented in dashed lines.

Usually, $\mathrm{Tl}$ and Th cannot be defined as linear functions of $\mathrm{Pl}$ and $\mathrm{Ph}$ (respectively, the power dissipation of eddy currents in the lower and upper susceptors), mainly because the heat losses of the susceptors are not proportional to their temperatures. In this case, we can only impose and control either $\mathrm{Tl}$ or $\mathrm{Th}$ and thus the thermal gradient would not be controlled.

\section{A. A manual generation of a thermal gradient}

The schematic diagram (Figure 1) shows two susceptors that are placed on each side of a studied sample. A temperature controller holds a setpoint with a High Frequency (HF) generator from Celes that supplies the induction coil. The eddy currents induced in the susceptors heat them up by joule effect. Thus, the two susceptors generate a thermal gradient across the sample. The temperature of the lower element $\mathrm{Tl}$ being regulated with a temperature controller, a variation of Th and the thermal gradient can be ensured by moving the mobile set along z (see Figure 2). For instance, we used an alumina test sample, $5 \mathrm{~mm}$ thick and $10 \mathrm{~mm}$ in diameter.

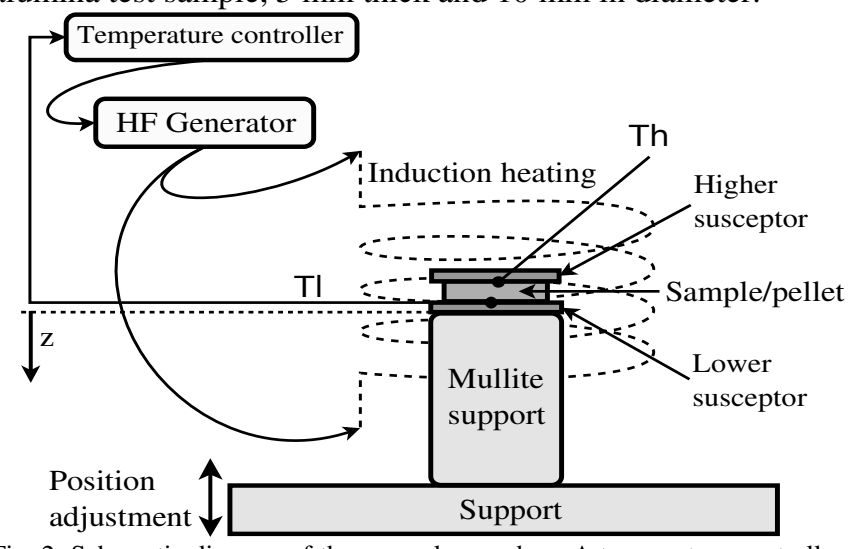

Fig. 2: Schematic diagram of the manual procedure. A temperature controller sets the 4-20 mA current loop with a high frequency (HF: $147 \mathrm{kHz}$, the frequency generated by our equipment) generator to reach the temperature setpoint Tl. A manual displacement of the mobile set makes it possible to vary Th and thus $\Delta \mathrm{T}$

After some manual experiments, for a given $\mathrm{z}$, we noticed that $\mathrm{Th}$ and $\Delta \mathrm{T}$ can be described as linear functions of $\mathrm{Tl}$. Also, for a given $\mathrm{Tl}$, the thermal differential $\Delta \mathrm{T}$ can be described as a linear function of $\mathrm{z}$. In the next subsections, we will explain why and in which conditions, this modeling can be used in a manual operation or in a computerized automated TGG, how to calibrate the setup and how to operate it 
manually, in order to generate a required thermal gradient.

\section{B. Proof of concept}

Let us assume that the two susceptors have much higher thermal conductivity than the sample (at least 10 times) and thus there is not a thermal interference between the two susceptors. Let us define two functions $\mathrm{Fh}$ and $\mathrm{Fl}$ such as:

$$
\left\{\begin{aligned}
T h & =F h \cdot P h+C_{1} \\
T l & =F l \cdot P l+C_{2}
\end{aligned}\right.
$$

Fh and $\mathrm{Fl}$ are mainly functions of geometry and the thermal losses of the susceptors. $\mathrm{C} 1$ and $\mathrm{C} 2$ are offsets (constants) originating from the influence of the ambient temperature. Consistently with (1), $\mathrm{Ph}$ and $\mathrm{Pl}$ are functions of a local peak magnetic field ( $\mathrm{Bh}$ and $\mathrm{Bl}$, respectively), the other parameters being the same for the two susceptors.

If the thickness of one susceptor is just a few times higher than the other's (but the two circumferences are at least 10 times higher than the two thicknesses), the thermal losses would be similar for the two, since the specific surface doesn't differ much (meaning that $\mathrm{Fh} \approx \mathrm{Fl}$ ). In these conditions and as $\mathrm{C}_{1}, \mathrm{C}_{2}, \mathrm{Tl}$, and $\mathrm{Th}$ are considered constant, we can write that:

$$
\frac{F h}{F l}=1 \rightarrow \frac{T h-C_{1}}{T l-C_{2}}=\frac{P h}{P l}=A
$$

The constant $A$ will depend mainly on the thicknesses of the two susceptors. Furthermore, if the thickness of the sample is, at least, 10 times smaller than the coil length, meaning that the two susceptors are very close to each other, $\mathrm{Bh}$ and $\mathrm{Bl}$ would be very similar and thus $A$ would have a value of 1 if the two susceptors have the same thickness and are made with the same material. From equation (3) and posing $\mathrm{C}_{1}-\mathrm{A} \cdot \mathrm{C}_{2}=$ To, we obtain:

$$
T h=A . T l+T o
$$

In the following section, we introduce our measurements that are carried out by manual displacements of the mobile set (the two susceptors and the sample) showing that this modeling is possible. Consequently, $\Delta \mathrm{T}$ can be modeled as:

$$
\Delta \mathrm{T}=\mathrm{Tl}-\mathrm{Th}=(1-\mathrm{A}) . \mathrm{Tl}-\mathrm{To}
$$

$\mathrm{Tl}$ being fixed by the temperature controller, since $\Delta \mathrm{T}$ can be modeled as a linear function of $\mathrm{Tl}$, we can also assume that, for a fixed position $\mathrm{z}$ :

$$
\left\{\begin{array}{l}
\Delta T=G l . T l+m_{1} \\
z=G h . T h+m_{2}
\end{array}\right.
$$

Where Gh and Gl are mainly functions of geometry and the heat losses of the susceptors. For the same reasons as for the previous case, and with the same conditions, we can write:

$$
\frac{G l}{G h}=1 \rightarrow \frac{\Delta T-m_{1}}{z-m_{2}}=\frac{T l}{T h}=k
$$

By posing $\mathrm{m}=\mathrm{m}_{1}-\mathrm{m}_{2} \cdot \mathrm{k}$, we obtain:

$$
\Delta T=k \cdot z+m
$$

It should be noted that a calibration is needed for each type of material used as samples and for any replacement of susceptors.

\section{Calibration measurements}

A first series of measurements made it possible to demonstrate a good linearity of Th as a function of $\mathrm{Tl}$ (with a fixed $\mathrm{z}$ position) in stabilized values, as well as good linearity of the differential $\Delta \mathrm{T}$ as a function of $\mathrm{z}$, at a fixed temperature
Tl. A second series of measurements has been undertaken to study the repetitiveness of the measurements. It confirmed the first series.

\section{a) Variation of the temperature $\mathrm{Tl}($ at $\mathrm{z}=0$ )}

As shown in Figure 3, the measurements allow a linear regression according to (4), with $\mathrm{A}=0.8275$ and $\mathrm{To}=71.31$.

The mobile set is slightly eccentric upwards, with respect to the coil. In this case, Th is lower than or equal to $\mathrm{Tl}$ (because the distance to the coil center is larger and the local magnetic field lower). Therefore, we have positive $\Delta \mathrm{Ts}$, as it is shown in Figure 4.

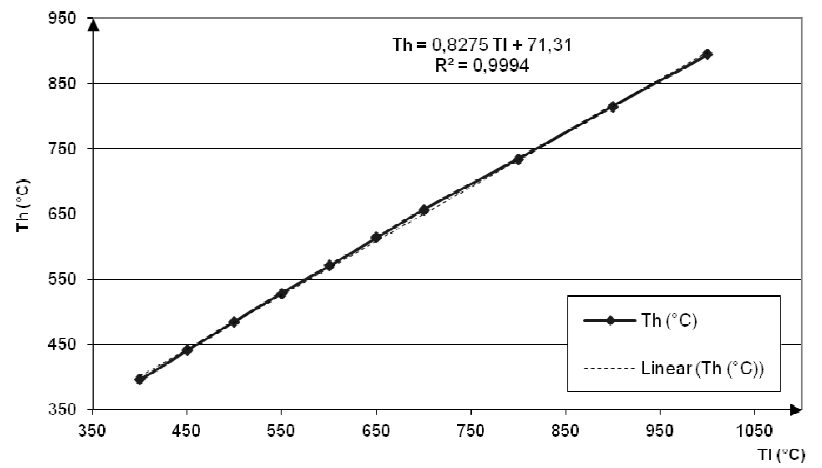

Fig. 3: Variation of Th as a function of Tl, with constant $\mathrm{z}$. The relationship is linear.

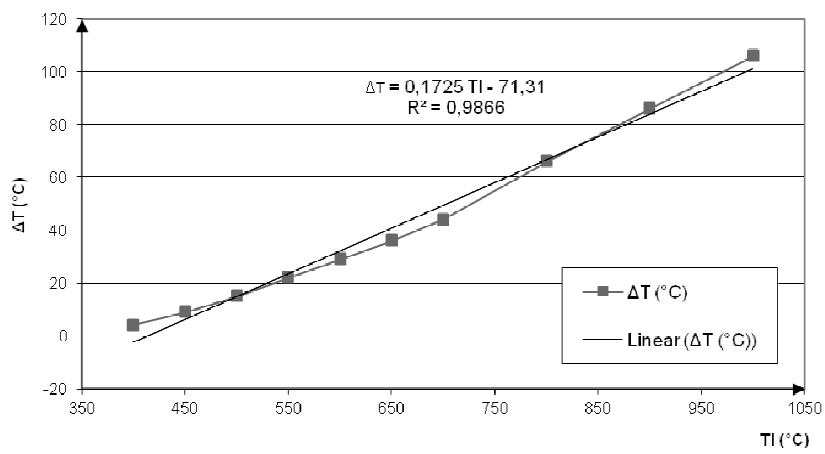

Fig. 4: Variation of $\Delta \mathrm{T}$ as a function of $\mathrm{Tl}$ : linear, in first approximation.

But, $\Delta \mathrm{T}$ depends also on $\mathrm{z}$ and should verify (8). Therefore, in the next section, we vary $\mathrm{z}$, keeping $\mathrm{Tl}$ constant at $700^{\circ} \mathrm{C}$.

b) Variation of the position $\mathrm{z}\left(\right.$ at $\mathrm{Tl}=700^{\circ} \mathrm{C}$ ).

Th varies linearly as a function of $\mathrm{z}$ (see Figure 5) and Figure 6 shows that $\Delta \mathrm{T}$ can be modeled linearly (according to equation 8), as a function of $\mathrm{z}$.

The parameter $\mathrm{k}$ is a displacement calibration constant. In our case: $\mathrm{k}=-3.86^{\circ} \mathrm{C} / \mathrm{mm}$ and $\mathrm{m}=56.9$ (see Figure 6).

At $700^{\circ} \mathrm{C}$, we can therefore have, at most, a gradient of $11^{\circ} \mathrm{C} / \mathrm{mm}$. This thermal gradient may increase with a more massive upper susceptor, and at higher temperatures.

c) Conditions of the measurements

It is necessary to wait thermal stabilization, a few minutes before taking into account the measurement ( 2 minutes, in our case). To check if the thermocouples can act as heating elements, we performed a heating cycle with only one thermocouple (without the mobile set): the thermocouple follows the set point (tested up to $150^{\circ} \mathrm{C}$ ). A fast heating of $+25^{\circ} \mathrm{C}$ is reached in about 3 seconds. Therefore, the thermal 
inertia of the thermocouple is low, which means that its temperature adapts to that of the susceptor.

The susceptors have a short response time but a low heat dissipation. For this reason we have chosen a set point rate (SPrr for a Eurothem controller) at $30^{\circ} \mathrm{C} / \mathrm{min}$ (i.e., $900^{\circ} \mathrm{C}$ reached in 30 minutes). The controller does not keep the set point in case of a power failure.

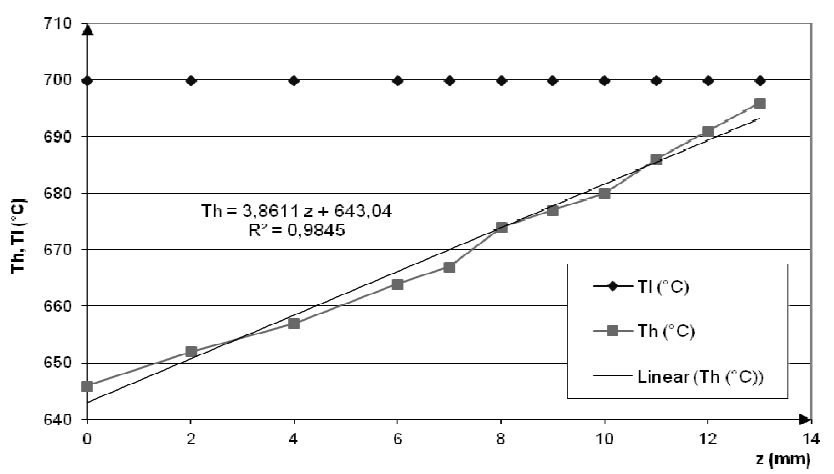

Fig. 5: Tl is kept constant in order to study Th as a function of the position $\mathrm{z}$.

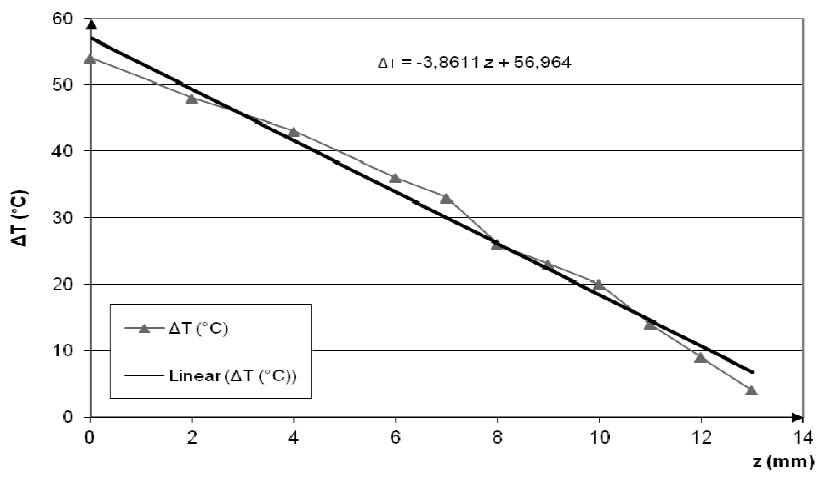

Fig. 6: The temperature difference $\Delta \mathrm{T}$ can be modeled as a function of the position $\mathrm{z}$.

\section{D.Manual control and operation}

Let $\mathrm{g}$ be the required thermal gradient $\left({ }^{\circ} \mathrm{C} / \mathrm{mm}\right)$ and $\mathrm{e}$, the thickness of the studied sample. A manual control is possible as long as the external disturbances are minimized.

a) Manual position adjustment for a required gradient $\mathbf{g}$

To adjust the position, we can calculate $\Delta \mathrm{T}=\mathrm{g}$.e, then determine $\mathrm{Tl}$ with equation (5). Finally, we calculate the position $\mathrm{z}$ to be reached in order to get the pair of set points $(\mathrm{Tl}, \Delta \mathrm{T})$, with equation (8):

b) Typical manual procedure

$$
Z=\frac{(\Delta T-m)}{k}
$$

According to our tests, in this configuration, we can reach a thermal gradient of up to $20{ }^{\circ} \mathrm{C} / \mathrm{mm}$ and a temperature of up to $1000^{\circ} \mathrm{C}$ on one of the two faces. The following procedure allows typically the application of a thermal gradient:

1- For a given material and thickness, calibrate the system by determining the equations (5) and (8).

2- The calibration phase being complete, place a new sample.

3- Choose $\Delta \mathrm{T}$, i.e. a gradient $\mathrm{g}$ given by $\mathrm{g}=\Delta \mathrm{T} / \mathrm{e}$.

4- With equation (9), determine and set the position $\mathrm{z}$. The response time of $\Delta \mathrm{T}$ is longer than the response time of $\mathrm{Tl}$.

5- Finally, determine $\mathrm{Tl}$ with equation (5) and set it as setpoint to the temperature controller.

\section{A COMPUTER CONTROLLED TGG}

The manual procedure has many limitations and makes it difficult to regulate both set points (Th and $\Delta \mathrm{T}$ ) simultaneously to set a gradient. Computer-controlled experimentation can enable the automation of the process. In addition, it has the following advantages:

a) Do measurements more precisely and save them in a file.

b) Make the experiments repeatable and reproducible.

c) Allow an automatic calibration (by a leap of the temperature, then of $\mathrm{z}$ ). This is very useful if the dimensions of the susceptors are changed or if samples of different sizes, shapes or compositions are used.

d) Allow a sudden stop of the heating and follow the temperatures $\mathrm{Tl}$ and $\mathrm{Th}$ (equivalent to a quenching).

A. Configuration in piloted mode with two instructions ( $T l, \Delta T)$

We use a Eurotherm 2404 temperature controller (K type thermocouple) with a serial communication module EIA 232 and a Modbus protocol. The temperature controller can thus be controlled by a PC and a LabView custom application. Figure 7 gives a general scheme of the experimental setup. The real temperature $\mathrm{Tl}$ (Process Value) is also read continuously by the PC. The types of thermocouples allowed are: J, K, L, R, B, N, T, S, and PL2. For the measurement of Th, an external data acquisition module (USB) is used (National Instruments: NI cDAQ 9172). A type K thermocouple is used as a sensor. The possible types of thermocouples are: J,K,N,R,S,T,B, and E.

For the control of the temperature differential $\Delta \mathrm{T}$, a motorized stage makes it possible to move the mobile set and thus to move along $\mathrm{z}$ to reach the desired $\Delta \mathrm{T}$. The motorized stage, controlled through a USB port, includes a stepper motor and its controller: Model L490MZ/M from ThorLabs. There are two ways to control this stage, one is absolute, and the other is relative. After some tests, it seems that its absolute control is more robust. Therefore, we chose this mode.

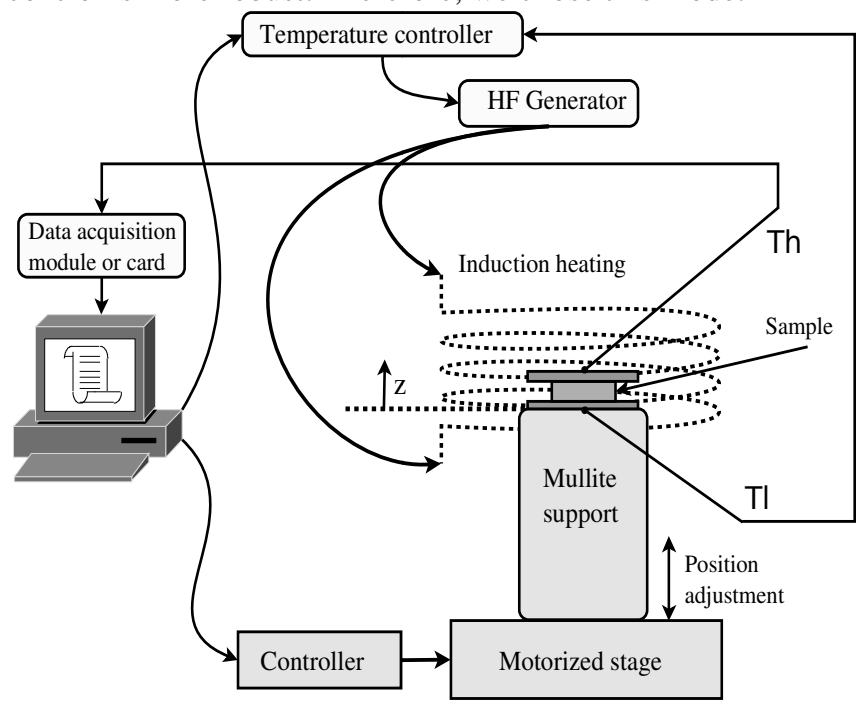

Fig. 7: Overall scheme of the experimental setup. The temperature controller sets Tl through the power of the HF generator while a motorized stage allows the control of $\Delta \mathrm{T}$ (and Th). 


\section{B. Optimization of susceptors}

We first tried platinum $(\mathrm{Pt})$ thin layers (by spreading platinum ink on ceramic substrates, then drying). After many tests, we noticed that the platinum layers eventually crack and no longer allow an efficient heating by eddy currents. For this reason, we opted for thin platinum plates that we had at our disposal (currently: $150 \mu \mathrm{m}$ and about $300 \mu \mathrm{m}$ thick for the lower and higher susceptors, respectively) of diameter widely greater than that of the sample (our samples are cylindrical pellets). Both Pt plates and PVD (Physical Vapor Deposition) deposited layers could be used to increase thermal contact.

\section{C.Mounting diagram}

In Figure 8, the mechanical setup is presented, showing the mobile set under the coil and the possibility of fixing $z_{o}$ (initial $\mathrm{z})$ with a preset wheel in $\mathrm{z}$.

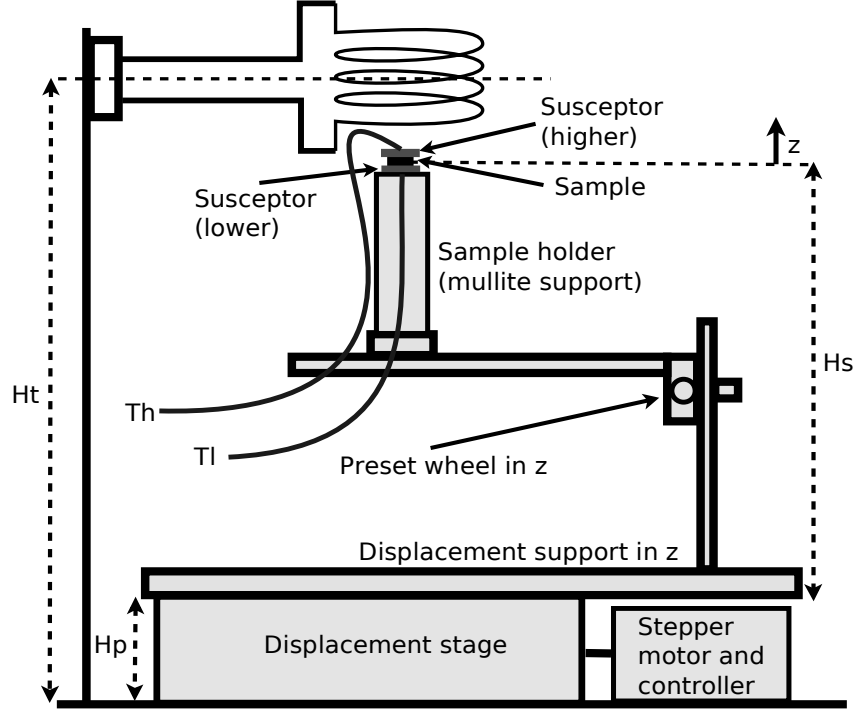

Fig. 8: Assembling diagram of the automatic TGG equipment.

In our device, $\mathrm{Hs}$ is between $330 \mathrm{~mm}$ and $400 \mathrm{~mm}$ and can be set with the preset wheel. We fix it once and for all at $\mathrm{Hs}=350 \mathrm{~mm}$. Let $\mathrm{Ht}$ be the height at which the axis of the induction coil must be placed, we need a withdrawal margin such as:

$$
\begin{aligned}
\mathrm{Ht} & =\mathrm{Hp}+\mathrm{Hs}+\text { Withdrawal margin } \\
& =58.5+350+40=448.5 \mathrm{~mm}(\text { we fix } \mathrm{Ht}=448 \mathrm{~mm}) .
\end{aligned}
$$

To have a good mechanical stability, the length of the $\mathrm{z}$ displacement support must be larger than the height of the sample holder and therefore, it is fixed at $325 \mathrm{~mm}$.

\section{Control}

Since the lower thermocouple is more likely to be in close contact (supporting the weight of the mobile set) than the top one, it is more secure to regulate $\mathrm{Tl}$ with the HF generator. If Th thermocouple is not in contact we risk having an unlimited rise of $z$. For this reason the maximum movement in the control application is limited to $50 \mathrm{~mm}$, which also corresponds to the maximum stroke of the motorized stage. In order to simplify the control, the rise and the decrease in temperature will be done with the same rate/slope.

Since the heat goes up, if the elements are introduced from the bottom of the coil to the top, we should reach higher temperature differentials. So we change the configuration, introducing the elements from the bottom of the coil. Thus, in the new configuration, we have :

$$
\begin{aligned}
& T h=a \cdot T l+T_{0} \\
& \Delta T=k^{\prime} \cdot z+m
\end{aligned}
$$

We want to regulate $\mathrm{Tl}$ and $\Delta \mathrm{T}$, at the same time. Note that To and $\mathrm{m}$ would not have the same values as before. They depend on the initial positions and temperatures.

Th will be higher than $\mathrm{Tl}$ and the roles of $\mathrm{Th}$ and $\mathrm{Tl}$ will be reversed. Only the slopes are to be kept (a second series of measurements would determine the new To and $m$ values). If we express $\mathrm{Tl}$ as a function of $\mathrm{Th}$ (from equation 4 ), the slope will be $\mathrm{a}=1 / \mathrm{A}=1 / 0.8275 \approx 1.2$. For the value of $\mathrm{k}$, approaching from the bottom ( $\mathrm{z}$ directed upwards), $\mathrm{k}$ would keep its value, so finally $k^{\prime}=k=-3.8611$. Thus, we have a new system of equations:

$$
\begin{aligned}
& \Delta T=T h-T l=(a-1) . T l+T o(\text { with } a>0), \\
& \Delta T=k \cdot z+m(k<0 \text { for } z \text { directed upwards }),
\end{aligned}
$$

where a and $\mathrm{k}$ are known, but To and $\mathrm{m}$ are to be determined as explained in the next section.

\section{E. Automatic calibration of the TGG}

We note that the more massive the higher susceptor is, the faster $T h$ rises and $\Delta \mathrm{T}$ is reached. This allows larger $\Delta \mathrm{T}$ values. The thermal inertia being higher, the increased mass should also yield less fluctuation. To calibrate the TGG completely (example: for a new type of sample), around an operating point, we can determine $\mathbf{k}, \mathbf{a}, \mathbf{m}$ and To by imposing a known leap in $\mathrm{z}$ and then a known leap of $\mathrm{Tl}$ :

- Calculation of $\mathbf{k}$ by a small variation of $\mathrm{z}$ :

$$
\begin{aligned}
& \text { at } \mathrm{z}_{1}: \quad \Delta \mathrm{T}_{1}=\mathrm{Th}_{1}-\mathrm{Tl}=\mathrm{k} \cdot \mathrm{z}_{1}+\mathrm{m} \\
& \text { at } \mathrm{z}_{2}: \quad \Delta \mathrm{T}_{2}=\mathrm{Th}_{2}-\mathrm{Tl}=\mathrm{k} \cdot \mathrm{z}_{2}+\mathrm{m} \\
& \Delta \mathrm{T}_{2}-\Delta \mathrm{T}_{1}=\mathrm{Th}_{2}-\mathrm{Tl}-\mathrm{Th}_{1}+\mathrm{Tl}=\mathrm{Th}_{2}-\mathrm{Th}_{1}=\mathrm{k} .\left(\mathrm{z}_{2}-\mathrm{Z}_{1}\right) \\
& \text { from where: } \mathbf{k}=\left(\mathrm{Th}_{\mathbf{2}}-\mathrm{Th}_{\mathbf{1}}\right) /\left(\mathbf{z}_{\mathbf{2}}-\mathrm{z}_{\mathbf{1}}\right) \\
& \text { - Calculation of } \mathbf{a} \text { by a small variation of } \mathrm{Tl} \text { : } \\
& \text { at } \mathrm{Tl}_{1}=\mathrm{T}: \quad \mathrm{Th}_{1}=\mathrm{a} . \mathrm{T}+\mathrm{To} \\
& \text { at } \mathrm{Tl}_{2}=\mathrm{T}+\mathrm{T}_{\mathrm{v}}: \quad \mathrm{Th}_{2}=\mathrm{a} .\left(\mathrm{T}+\mathrm{T}_{\mathrm{v}}\right)+\mathrm{To} \\
& \mathrm{Th}_{2}-\mathrm{Th}_{1}=\mathrm{a} . \mathrm{T}_{\mathrm{v}}, \\
& \text { from where: } \mathbf{a}=\left(\mathbf{T h}_{\mathbf{2}}-\mathbf{T h}_{\mathbf{1}}\right) /\left(\mathbf{T l}_{\mathbf{2}}-\mathbf{T l}_{\mathbf{1}}\right)
\end{aligned}
$$

- Calculation of $\mathbf{m}$ and To to regulate $\Delta \mathrm{T}$ :

We checked that for different Tl leaps, $\mathbf{k}$ is constant and for different z leaps, a is also constant. However, $\mathbf{m}$ and To depend on the initial temperature (ambient) and position. We choose a set point (e.g., $\mathrm{z}=0, \mathrm{Tl}=200^{\circ} \mathrm{C}$ ). With the equation (10), we calculate $\mathrm{To}=\mathrm{Th}-\mathrm{a} . \mathrm{Tl}$. Then, with the equations (12) and (13), we calculate:

$$
m=\Delta \mathrm{T}-\mathrm{k} \cdot \mathrm{z}=(\mathrm{a}-1) \cdot \mathrm{Tl}+\mathrm{To}-\mathrm{k} \cdot \mathrm{z}
$$

These four parameters can be determined manually, but we also integrated these automatic procedures in our dedicated Labview application. It should be noticed that an optimal velocity of the stage should be chosen (a low value yields fluctuations on $\Delta \mathrm{T}$ and a high value leads to a permanently turning motor).

We want a small variation $\delta \Delta \mathrm{T}$, due to perturbations, to be compensated by a small displacement in $\delta z$, hence, after derivation, we obtain from the equation (13), the relation: 


$$
\delta z=\frac{1}{k} \delta \Delta T
$$

This relation is equivalent to a PI (Proportional, Integral) controller which is enough stable in our setup, where the proportional part is null (this is due to the fact that we have a progressively rising or decreasing setpoint).

\section{F. Optimization of the temperature controller parameters}

After various tests with our temperature controller, the following PID parameters gave the best results:

$$
\mathrm{Pd}=6, \mathrm{ti}=13, \mathrm{td}=2 \text {, }
$$

where, $\mathrm{Pd}$, ti, and td are respectively the proportional, integral and derivative coefficients. For the regulation of $\Delta \mathrm{T}$, an acceleration of $0.25 \mathrm{~mm} / \mathrm{s}^{2}$ and a velocity of $0.5 \mathrm{~mm} / \mathrm{s}$ are the best motorized stage parameters. A new calibration was done at $300^{\circ} \mathrm{C}$ and gave the following parameters for the TGG:

$a=1.58 \mathrm{k}=-1.06^{\circ} \mathrm{C} / \mathrm{mm}$ To=-95.3 ${ }^{\circ} \mathrm{C}$ and $\mathrm{m}=78.4^{\circ} \mathrm{C}$.

Note: it is logical that the achievable (maximal) $\Delta \mathrm{T}$ depends on the chosen $\mathrm{Tl}$.

\section{CONTROL SOFTWARE}

We designed and built up a dedicated user-friendly LabView application to control the experimental setup. The software environment is as follows: a PC for a development carried out on Windows XP and a LabView license (version 8.6, Full development). The developed application (see Figure 9) saves some values each second (or with a selected period) during an automatic thermal gradient generation with a chosen temperature profile. These values are: Time, Th, Tl, $\Delta \mathrm{T}, \mathrm{z}$ and the phase (increase, decrease, dwell) represented by respectively by ("M", "D" and "-_").

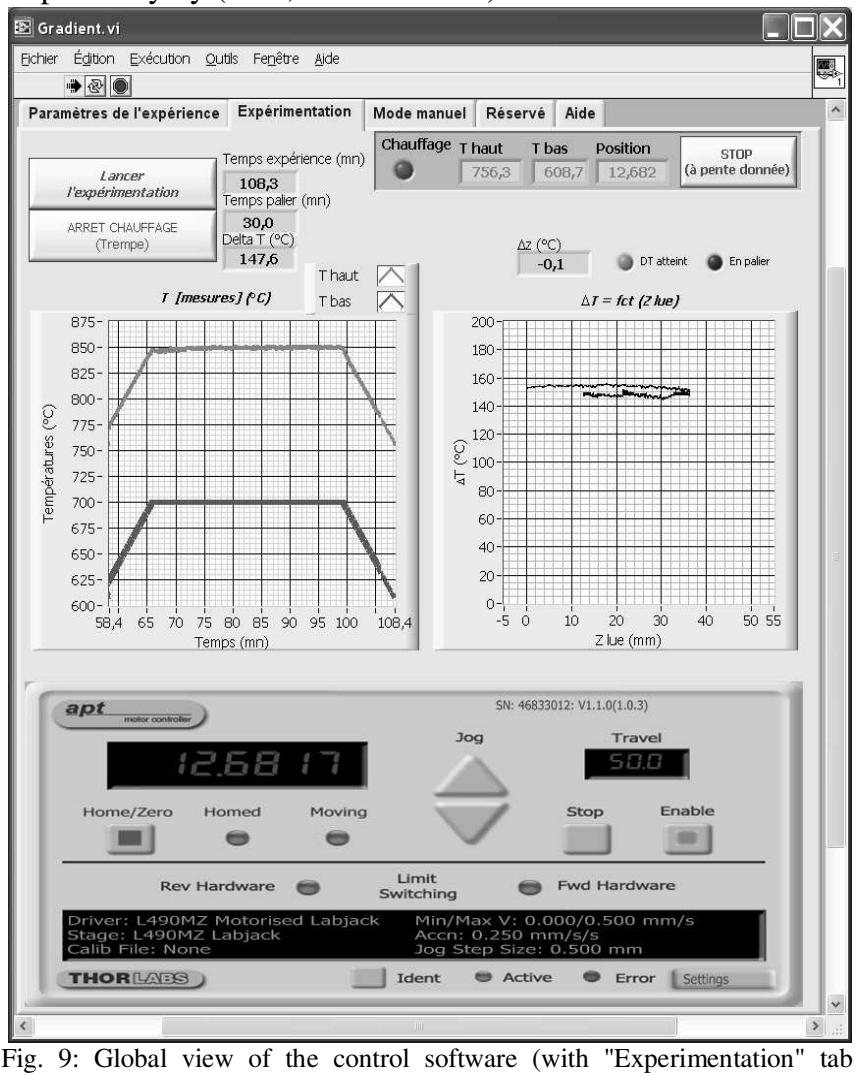
selected).
The different tabs of the "Gradient" application are: "Parameters", "Experimentation", "Manual Mode", "Reserved" and "Help"). This control software is developed in LabView 8.6 graphical language. In the "Reserved" tab, there is, in particular, the possibility of recalibrating the system (determining a, $\mathrm{k}$, To and $\mathrm{m}$ ).

One can see the different tabs during the experimentation. Some parameters, such as dwell time, Th and therefore $\Delta \mathrm{T}$ can be changed throughout the ongoing experiment, if necessary. A certain number of commands and measured values are available in all the tabs (e.g., Th, Tl, action on $\mathrm{z}$ ) to be able to follow the experiment at any time and react as quickly as possible, if necessary.

Figure 10 shows a general view of the equipment, while Figure 11 shows the top views of the coil and the mobile set during experiments at two different temperatures.

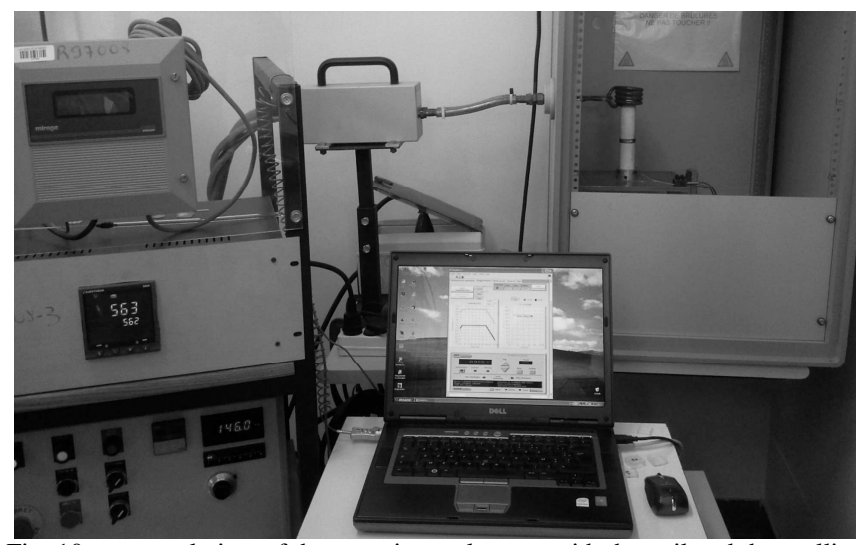

Fig. 10: a general view of the experimental setup, with the coil and the mullite support seen in the upper-right corner (in red and white, respectively).
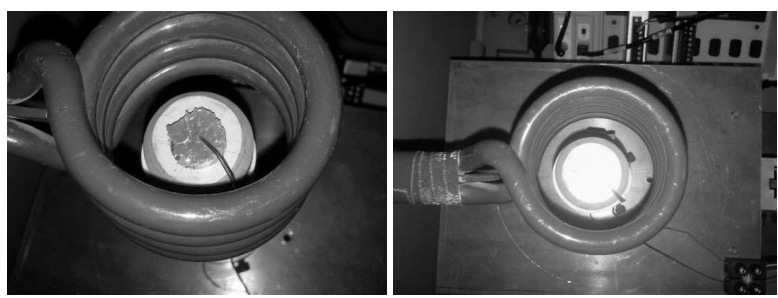

Fig. 11: Photographs during experimentations. Left and right pictures: the higher susceptor and thermocouple can be seen at around $750^{\circ} \mathrm{C}$ and $800^{\circ} \mathrm{C}$, respectively. The susceptor appears white in the right picture. This is due to a wider emission spectrum.

\section{RESULTS AND VALIDATION}

Since platinum plates are used, cracks can be avoided and higher slopes (temperatures rates) scheduled. Three platinum disks (diameter: $14 \mathrm{~mm}$, thickness: $0.15 \mathrm{~mm}$ ) were cut in a platinum plate. Two contiguous discs, one on the other, were used as upper (higher) susceptor and one disc on the ceramic support as lower susceptor.

\section{A. Gradient with simulation of a "quench" (alumina sample)}

This experiment was done at an early stage of development, where the parameters (To, k, and $\mathrm{m}$ ) where not well calibrated. Therefore, Th is not rigorously stabilized during the $580^{\circ} \mathrm{C}$ dwell.

An automatic adjustment of the $\mathrm{Tl}$ regulator was done and gave the following parameters: $\mathrm{Pd}=194, \mathrm{ti}=16$, $\mathrm{td}=2$. Then, 
an automatic calibration of the system was done at $250^{\circ} \mathrm{C}$ and it gave the following parameters: $\mathrm{a}=1.21, \mathrm{To}=-40.4^{\circ} \mathrm{C}, \mathrm{k}=-$ $0.519^{\circ} \mathrm{C} / \mathrm{mm}, \mathrm{m}=13.7^{\circ} \mathrm{C}$. The regulation in $\mathrm{z}$ was carried out with the following motorized stage parameters: acceleration $=0.25 \mathrm{~mm} / \mathrm{s}^{2}$, velocity $=0.5 \mathrm{~mm} / \mathrm{s}$.

An experiment at $\left(\mathrm{Th}=580^{\circ} \mathrm{C}, \mathrm{Tl}=550^{\circ} \mathrm{C}\right.$, slope $\left.=20^{\circ} \mathrm{C} / \mathrm{min}\right)$ was carried out on a $5 \mathrm{~mm}$ thick alumina sample. It is presented in Figure 12 (started at about $500^{\circ} \mathrm{C}$ ).

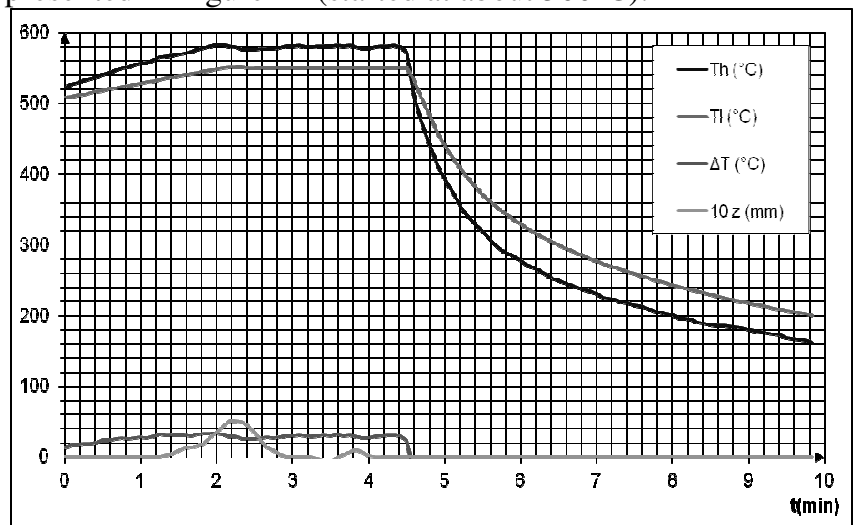

Fig. 12: An experiment with a "quenching". Note that, immediately after a quench, Th becomes lower than Tl because of its higher surface of contact with the air and its low thickness.

Before "quenching", the chosen rate is high $\left(20^{\circ} \mathrm{C} / \mathrm{min}\right)$, then the variations around the instructions (setpoints) are important. At $t=4.2$ min., the heating was stopped abruptly. Th ends with a value lower than $\mathrm{Tl}$. This is due to the fact that the lower element has a higher thermal inertia (this was done in early development, when the lower susceptor was thicker).

\section{B. Gradient with an imposed duration (semiconducting oxide ceramic sample)}

After adjusting the PID of the $\mathrm{Tl}$ regulator and a calibration of the system, an experiment at $\left(\mathrm{Th}=850^{\circ} \mathrm{C}, \mathrm{Tl}=700^{\circ} \mathrm{C}\right.$, slope: $10^{\circ} \mathrm{C} / \mathrm{min}$ ) was conducted on a semiconducting oxide ceramic sample, $2 \mathrm{~mm}$ thick and $9 \mathrm{~mm}$ in diameter. It is presented in Figure 13 (experiment started at room temperature).

For information, at around $\mathrm{Tl}=80^{\circ} \mathrm{C}$ (and even before) the frequency of the HF generator is stabilized at $145.9 \mathrm{kHz}$.

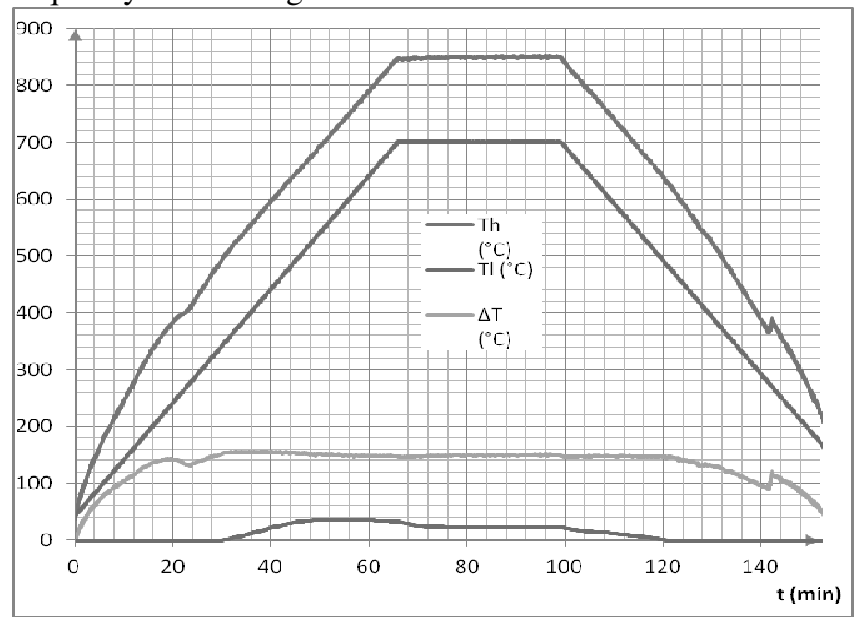

Fig. 13: An example of experiment, where it can be seen that for Th temperatures higher than $500^{\circ} \mathrm{C}$, the temperatures and thus the $\Delta \mathrm{T}$ are very well maintained. $\mathrm{Th}, \mathrm{Tl}, \Delta \mathrm{T}$, and $\mathrm{z}$ are saved during the whole experiment.

\section{CONCLUSIONS}

With the current setup, we generated thermal gradients under the following conditions: Th was raised up to $1000^{\circ} \mathrm{C}$ with $\Delta \mathrm{T}$ temperature differentials raised up to $150^{\circ} \mathrm{C}$ for a 2 $\mathrm{mm}$ thick sample (which results in a thermal gradient of $75^{\circ} \mathrm{C} / \mathrm{mm}$ ). The samples had a thickness of 2 to $5 \mathrm{~mm}$ and low thermal conductivity (alumina: $6.2 \mathrm{~W} \cdot \mathrm{m}^{-1} \cdot \mathrm{K}^{-1}$ at $1000^{\circ} \mathrm{C}$, oxide ceramic: considered lower than that of alumina).

We are currently using this nondestructive testing equipment to study the ionic migration, previously evidenced in LAMOX oxide-ion conductors (see Lacorre et al. [9], Selmi et al. [10]). The capabilities quoted above are sufficient for our purpose. For other applications, the conditions of use could be modified. These conditions will depend on the materials to be studied (thermal conductivity, sample shape, etc.) and simple adaptations that can be done (e.g., differences in thickness between the two platinum plates).

Most of the possible applications are in research and development laboratories where, for instance, the diffusion of chemical species could be studied. Other studies and characterizations could involve the behavior of materials under fire, the behavior of composite materials and building materials submitted to thermal gradients. The TGG could also submit protection tiles and thermal insulators to known and controlled gradients for aging studies.

A modified version of this TGG could be used whenever it is necessary to create a steady-state heat flux in one or two directions, e.g., for conductivity measurements in a vacuum chamber. According to Zhang et al. [11], the alternating directions, easily carried out with our setup, allows better thermal conductivity measurements. Zawilski et al.[12] stress that for small samples (e.g., in thickness), it is difficult to fix sensors on the sample, thus a global thermal conductance measurement, consistent with our setup, is better suited.

\section{ACKNOWLEDGMENT}

The authors would like to thank Pierre Rabadeux who performed some preliminary tests on susceptor materials, and the organization SATT Ouest-Valorisation (Rennes, France), for their help with a patent application and further development and valorization prospects.

\section{REFERENCES}

[1]D. Shin and H.I. Yoo, "Oxygen thermomigration in acceptor-doped perovskite," Phys. Chem. Chem Phys., vol. 19, no. 18, pp. 11120-11130, 2017, 10.1039/c7cp00384f.

[2]C.J. Metting et al., "Note: A simple thermal gradient annealing unit for the treatment of thin films," $R$. Scientific Instruments, vol. 84, no. 3, pp. 17701774, March 2013, 10.1063/1.4795831.

[3]M. Bober, C. Sari, G. Schumacher, "Redistribution of plutonium and uranium in mixed (U, Pu) oxide fuel materials in a thermal gradient," $J$. Nuclear Materials, vol. 39, no. 3, pp. 265-284, June 1971, 10.1016/00223115(71)90146-2.

[4]Device for generating a high temperature gradient in a sample comprising optical monitoring means, by Y. Pontillon, E. Hanus, H. Capdeliva. (2015, Apr. 9). Patent US 2015/0098485 A1. [Online].

[5]S. Shihab, L. Thevenard, A. Lemaître, J.Y. Duquesne, C. Gourdon, "Steady-state thermal gradient induced by pulsed laser excitation in a ferromagnetic layer," J. Applied Physics, vol. 119, no. 15, pp. 153904, Apr. $2016,10.1063 / 1.4947226$ 
[6] Temperature gradient system and method, by R.W. Obbard, N.P. Afonina, R. Lieb-Lappen, C.G. Pope. (2016, June 23). Patent US 2016/0178264 A1. [Online].

[7] K.C. Liu, "Ceramic Specimen Heating by Induction Power," JInt. J High Technology Ceramics, vol. 4, no. 2-4, pp. 203-210, 1988, 10.1016/02673762(88)90045-8.

[8]L. Nickelson, Electromagnetic Theory and Plasmonics for Engineers. Singapore: Springer, 2019. Available: https://doi.org/10.1007/978-981-132352-2. Accessed on: Sept. 8, 2020.

[9]P. Lacorre, F. Goutenoire, O. Bohnke, R. Retoux, Y. Laligant, "Designing fast oxide-ion conductors based on La2Mo2O9", Nature, vol. 404, pp. 856858, Apr. 2000, 10.1038/35009069.

[10] A. Selmi, C. Galven, G. Corbel, P. Lacorre, "Thermal stability of alkali and alkaline-earth substituted LAMOX oxide-ion conductors", Dalton Trans., vol. 39, no. 1, pp. 93-102, Jan. 2010, 10.1039/b915080c.

[11] P. Zhang, B. Shi, Y. Xuan, Q. Li, "A high-precision method to measure thermal conductivity of solids using reversible heat flux," Meas. Sci. Technol., vol. 24, no. 9, July 2013, 10.1088/0957-0233/24/9/095004

[12] B.M. Zawilski, R.T. Littleton, T.M. Tritt, "Description of the parallel thermal conductance technique for the measurement of the thermal conductivity of small diameter samples," R. Scientific Instruments, vol. 72, no. 3, pp. 1770-1774, March 2001, 10.1060/1.1347980.

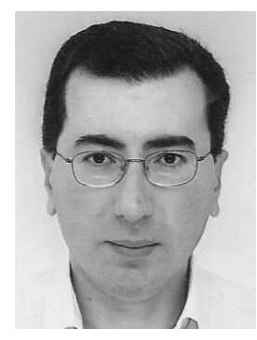

Veyis Gunes received an Engineering degree in electrical and electronics from ENSISA, Mulhouse, France, in 1994, the M.Sc degree in instrumentation and computer science for images, from the University of Burgundy, Dijon, France, in 1997, and a PhD degree in Computer Science (pattern recognition) from $\mathrm{La}$ Rochelle University, France, in 2001.

He was an Assistant-Professor at MIME Institute, Le Mans University (France) in 2001-02 and at IUT (University Institute of Technology, Electrical department) of Troyes (France) in 2002-2003. From 2005 to 2010, he worked on instrumentation projects involving mechanical properties and tribology at the University of Franche-Comté, France.

He is currently involved in instrumentation projects dealing with characterization and elaboration of new materials, at the Institute of Molecules and Materials of Le Mans University, France. His main research interests are instrumentation, electronics, material characterization, and machine learning.

Dr. Gunes was a recipient of an Innovation Award from Thomson Tubes and Displays (Genlis, France) in 1997 and he was an invited reviewer for IEEE Trans. Fuzzy Sets in 2004.

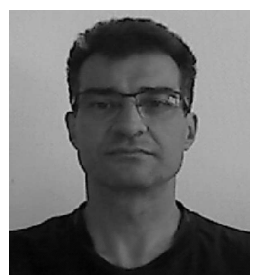

Jean-Yves Botquélen received a B.Eng. in Scientific Instrumentation from Conservatoire National des Arts et Métiers (CNAM, Paris, France), in 2016. He was a CNRS Technician at Laboratory of Oxides and Fluorides from 2001 to 2019, in Le Mans University, France. Since 2020, he has been an Engineer Assistant at the Institute of Molecules and Materials (IMMM), in the same university. While working on various instrumentation projects, he is also in charge of maintenance for different synthesis and analysis devices of the institute.

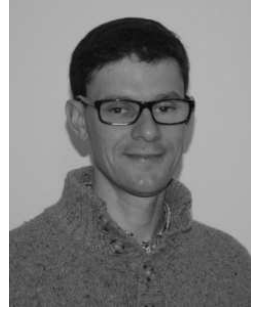

Gwenaël Corbel received the $\mathrm{PhD}$ in Materials Science and Solid State Chemistry and the accreditation to supervise research (Habilitation à Diriger les Recherches) from Le Mans University (France) in 1999 and 2017, respectively. In 1999-2001, he was a Marie-Curie research fellow at the Department of Chemistry from the University of Cambridge (United Kingdom). In 2001-2002, he was an Assistant-Professor at the Laboratory of Solid Crystallochemistry from Pierre and Marie Curie University in Paris (France). Since 2002, he is full-time junior CNRS researcher (Chargé de Recherche CNRS) at Le Mans University (France). He worked first in the Laboratory of Oxides and Fluorides until its full merger in 2012 with all chemistry and physics laboratories from Le Mans University (France) to form the Institute of Molecules and Materials of Le Mans (IMMM, UMR CNRS 6283). His research activities target the synthesis and structural characterization of fluorides, hydroxides, oxides and mixed anions compounds. The motivation for all studies is to relate the structural features of compounds to their physical (luminescence, magnetic, ionic conductivity) and bactericidal properties.

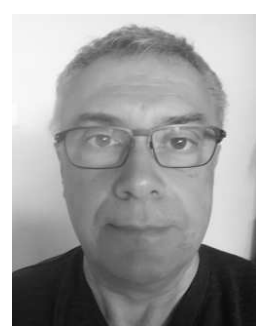

Philippe Lacorre received the Engineering degree in Materials and Chemistry from ENSICAEN (ISMRA, Caen, France) in 1981, and the $\mathrm{PhD}$ in Physics and Chemistry of Materials and the Accreditation to Supervise Research (HDR) from Le Mans University (France) in 1988 and 1997, respectively.

A researcher at the French Centre National de la Recherche Scientifique (CNRS) from 1985, he was detached at Institut Laue Langevin (ILL Grenoble, France) between 1985 and 1988, and at IBM Almaden Research Center (San José, California, USA) in 1989-90. Since 1997, he has been a CNRS Senior Researcher at Laboratoire des Oxydes et Fluorures, now Institut des Molécules et Matériaux du Mans (IMMM) in Le Mans University, France. His research interests include the synthesis, structure and structure-properties relationships of rare-earth transition metal oxides with magnetic, insulator-metal or ionic conductivity properties.

Dr. Lacorre was a recipient of the CNRS Bronze Medal (1990), of the Solid State Chemistry Award of the French Chemical Society (1993), and of the Jecker Price of the French Academy of Sciences (1998). 